\title{
Editorial
}

\section{Neurodynamic System Theory and Applications}

\author{
Jinde Cao, ${ }^{1,2}$ Xuerong Mao, ${ }^{3}$ and Qi Luo ${ }^{4}$ \\ ${ }^{1}$ Department of Mathematics, Southeast University, Nanjing 210096, China \\ ${ }^{2}$ Department of Mathematics, King Abdulaziz University, Jeddah 21589, Saudi Arabia \\ ${ }^{3}$ Department of Mathematics and Statistics, University of Strathclyde, Glasgow G1 1XH, UK \\ ${ }^{4}$ School of Information \& Control, Nanjing University of Information Science \& Technology, Nanjing 210044, China
}

Correspondence should be addressed to Jinde Cao; jdcao@seu.edu.cn

Received 12 June 2013; Accepted 12 June 2013

Copyright (C) 2013 Jinde Cao et al. This is an open access article distributed under the Creative Commons Attribution License, which permits unrestricted use, distribution, and reproduction in any medium, provided the original work is properly cited.

Neurodynamical systems have gradually become a popular research topic owing to their broad applications in such fields as optimization, linear and nonlinear programming, associative memory, pattern recognition, and computer vision. In view of some inevitable factors, there have been formed various neurodynamical systems including delayed neural networks, stochastic neural networks, impulsive neural networks, reaction-diffusion neural networks, and fuzzy neural networks. Over the last few decades, considerable attention has been devoted to this research area not only for enriching the theory of differential equations and dynamical systems but also for deeply understanding the dynamic states of neural networks for better modelling the brain.

The current special issue puts its emphasis on the study of neurodynamical system theory and applications. Call for papers has been carefully prepared by the guest editors and posted on the journal's web page, which has received many attentions followed by some submissions among wide topics such as delayed neural systems, stochastic neural systems, impulsive neural systems, reaction-diffusion neural systems, fuzzy neural systems, evolutionary neural systems, mathematical modeling of neural systems, computational neuroscience, neurodynamical optimization and adaptive dynamic programming, cognitive models, pattern recognition, and neural network applications.

All manuscripts submitted to this special issue went through a thorough peer-refereeing process. Based on the reviewers' reports, eleven original research articles are finally accepted. The contents embrace the synchronization of coupled neural networks, the numerical analysis of stochastic delayed partial differential equations, and the stability analysis of delayed impulsive reaction-diffusion neural networks and switched neural networks.

It is certainly impossible to provide in this short editorial a more comprehensive description for all articles in this special issue. However, the team of the guest editors believes that the results included reflect some recent trends in research and outline new ideas for future studies of neurodynamical system theory and applications.

\section{Acknowledgments}

We would like to express sincere gratitude to the authors who submitted papers for consideration and the many reviewers whose comments are important for us to make the decisions. All the participants have made it possible to have a very stimulating interchange of ideas. Many thanks are also given to the editorial board members of this journal owing to their great support and help for this special issue.

Jinde Cao

Xuerong Mao

Qi Luo 


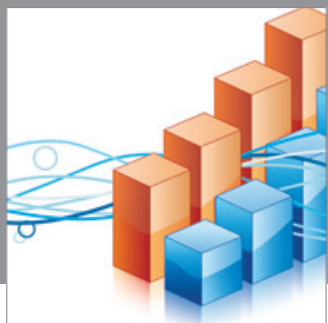

Advances in

Operations Research

mansans

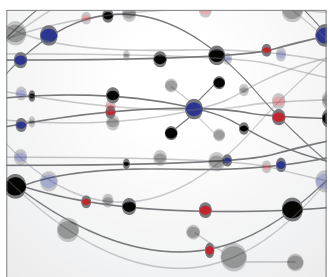

The Scientific World Journal
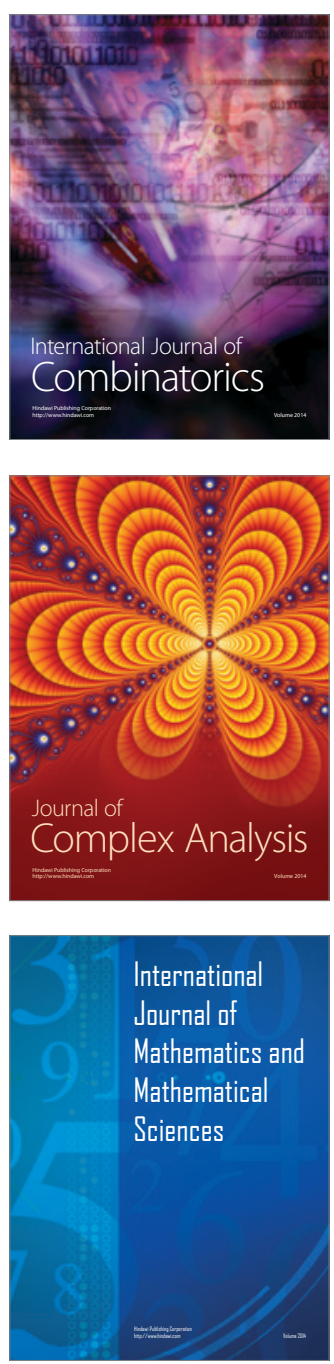
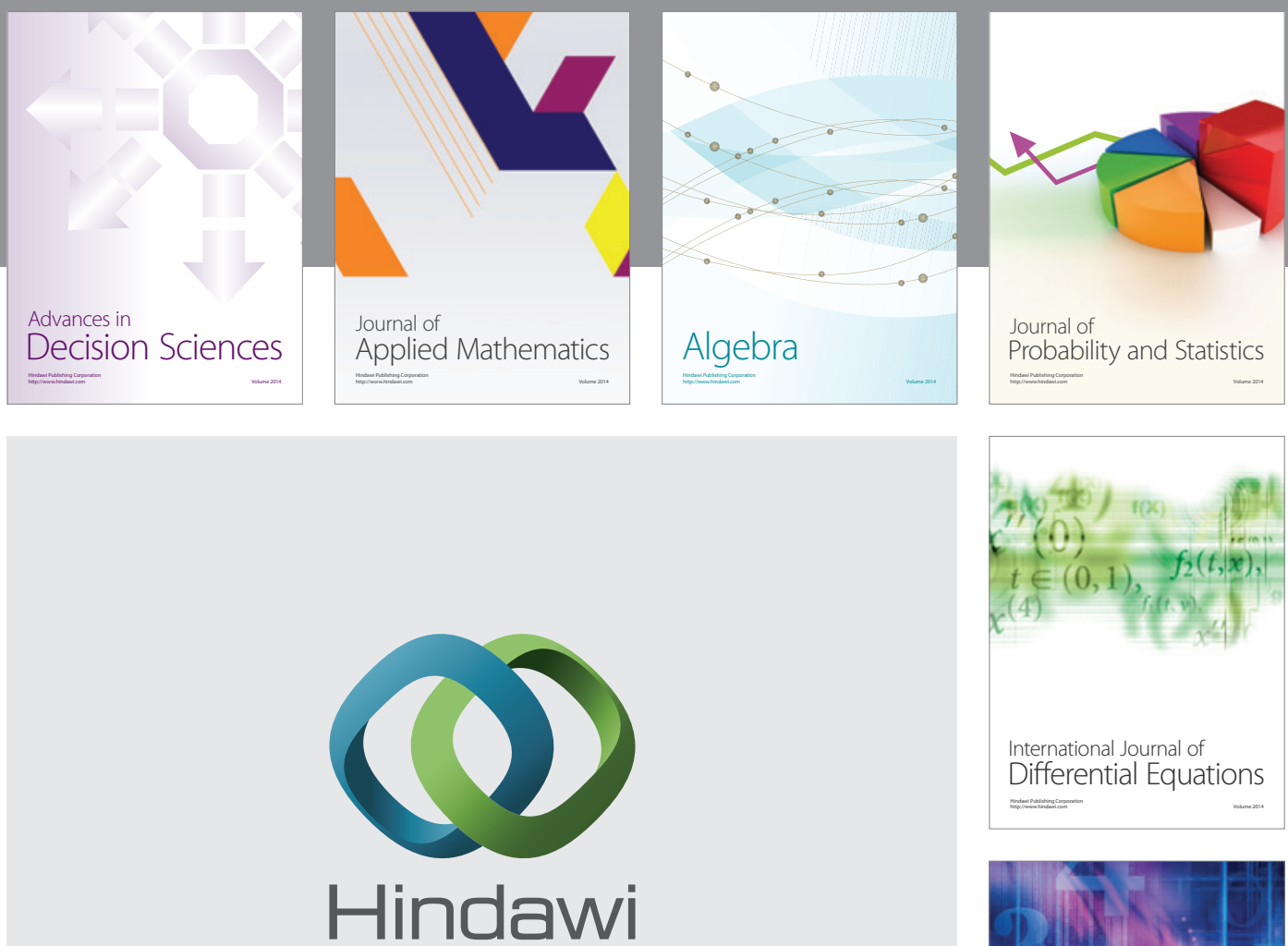

Submit your manuscripts at http://www.hindawi.com
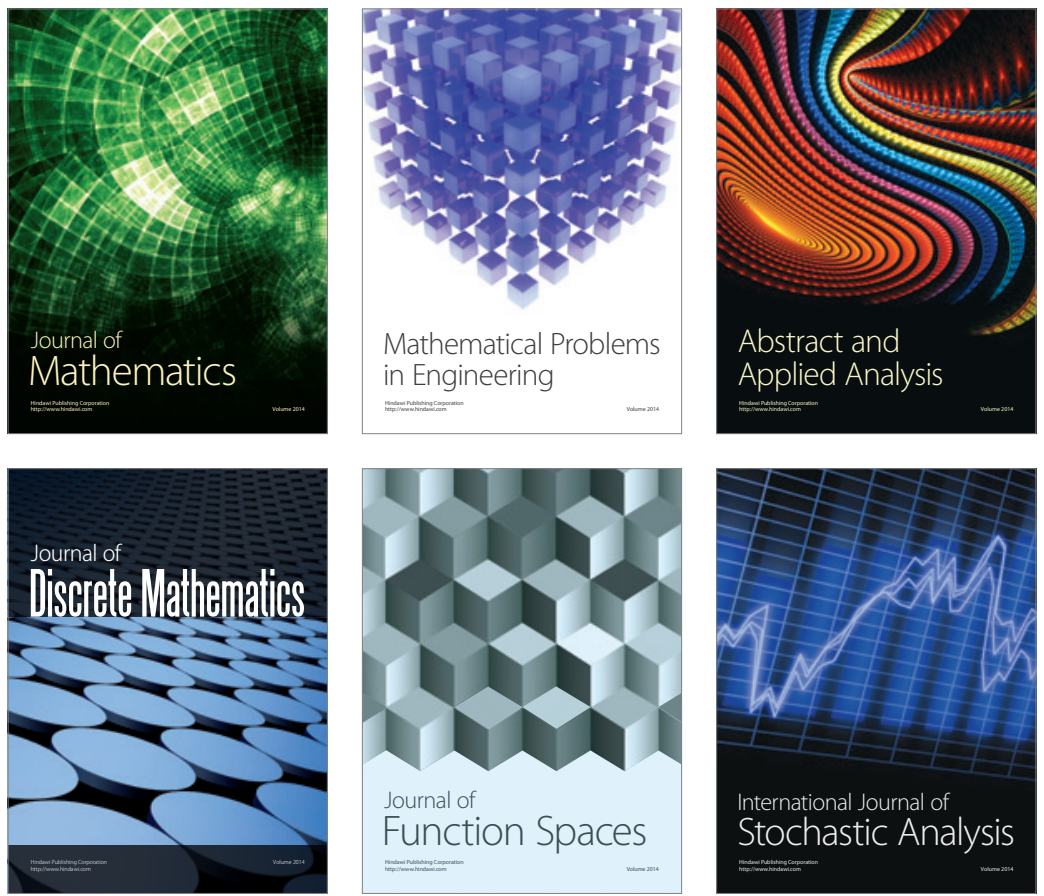

Journal of

Function Spaces

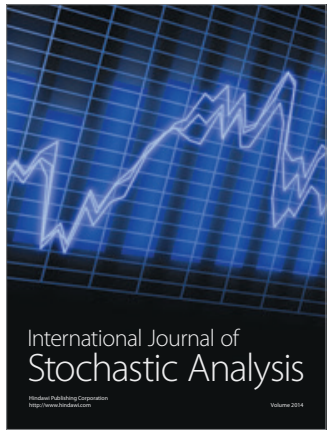

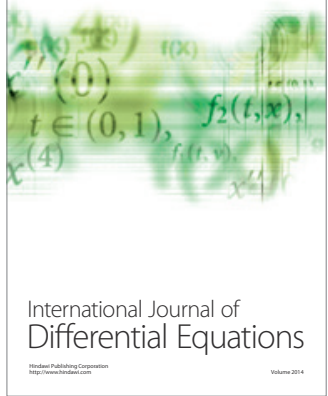
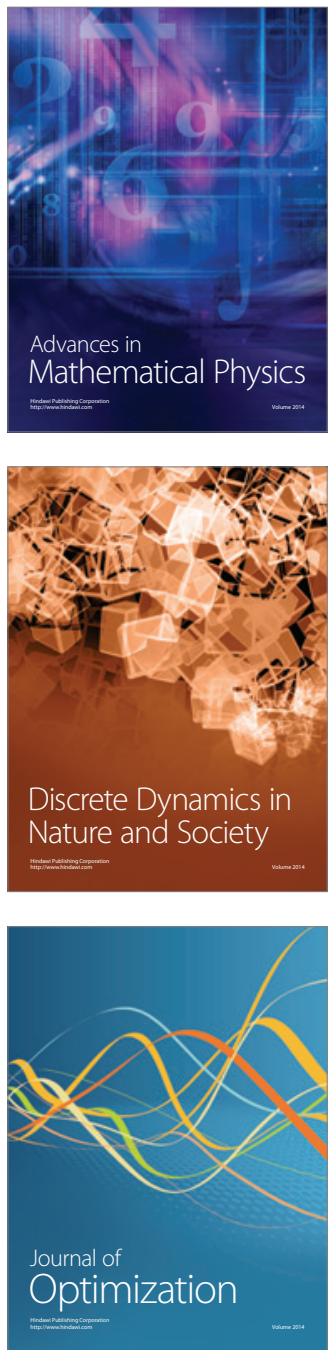